Alfonso-Mora, M.L.; Castellanos Garrido, A.L.; Nieto Rodríguez, J.F.; Sánchez Baquero, H.A.; Táutiva Cárdenas, L.D. (2019) Foot Dynamics in Balance, Power, Speed, and Tennis Service Tests. Revista Internacional de Medicina y Ciencias de la Actividad Física y el Deporte vol. 19 (75) pp. 387$397 \mathrm{Http}: / /$ cdeporte.rediris.es/revista/revista75/artdinamica1051.htm

DOI: 10.15366/rimcafd2019.75.001

\title{
ORIGINAL
}

\section{DINAMICA PLANTAR EN PRUEBAS DE BALANCE, POTENCIA, VELOCIDAD, Y SERVICIO EN TENISTAS}

\section{FOOT DYNAMICS IN BALANCE, POWER, SPEED, AND TENNIS SERVICE TESTS}

\author{
Alfonso-Mora, M.L.'; Castellanos Garrido, A.L.'; Nieto Rodríguez, J.F.2; \\ Sánchez Baquero, H.A. ${ }^{2}$ y Táutiva Cárdenas, L.D. ${ }^{2}$ \\ 1 Profesora Universidad de La Sabana Facultad de Enfermería y rehabilitación Fisioterapia. \\ (Colombia) margarethalmo@unisabana.edu.co, adriana.castellanos@unisabana.edu.co \\ ${ }^{2}$ Fisioterapeuta Universidad de La Sabana (Colombia) juannierod@unisabana.edu.co, \\ hectorsaba@unisabana.edu.co, laurataca@unisabana.edu.co.
}

\section{AGRADECIMIENTOS}

Los autores agradecen a la Universidad de La Sabana y al club deportivo Laverdieri.

Código UNESCO / UNESCO code: 3210 Otras especialidades Médicas Clasificación Consejo de Europa / Council of Europe classification: 3 Biomecánica del deporte / Biomechanics of sport

Recibido 18 de octubre de 2017 Received October 18, 2017 Aceptado 17 de diciembre de 2017 Accepted December 17, 2017

\section{RESUMEN}

El objetivo del presente reporte es describir la dinámica plantar durante el servicio y durante la realización de pruebas específicas de potencia, velocidad y equilibrio en tenistas y establecer su relación. Se estableció un método descriptivo, se evaluaron a 16 deportistas realizando pruebas de potencia, velocidad, equilibrio y servicios tipo top spin, se realizaron medidas descriptivas para los resultados de las pruebas y los de la dinámica plantar, y se correlacionaron entre sí. Los resultados sugieren que el centro de presión anteroposterior del pie (COP-AP) durante las pruebas de equilibrio tuvieron un promedio de $-32 \mathrm{~mm}$, a su vez, el promedio de potencia durante el salto en el servicio fue de $165 \pm 35 \mathrm{~N}$. Se encontró una relación $(r=0,45)$ entre el 
tiempo en el aire y los centímetros saltados, además entre el tiempo de la prueba de velocidad y el tiempo del ciclo de la carrera $(r=0,54)$.

PALABRAS CLAVE: Dinámica, pie, balance, potencia, velocidad, tenis, fisioterapia.

\section{ABSTRACT}

The objective of this article is to describe the foot dynamics during the service and during the performance of specific tests of power, speed and balance in tennis players and to establish their relationship. A descriptive method was established and 16 athletes were evaluated in power, velocity, balance and top spin services tests. Descriptive measures were performed for the test results and for foot dynamics, and correlated with each other. The results suggest that the center of anteroposterior foot pressure (COP-AP) during the balance tests had an average of -32 , the average power during the jump in the service was $165+35$. A relation ( $r$ $=0.45$ ) was found between the time in the air and the centimeters jumped, in addition, between the time of the test of speed and the time of the cycle of the race $(r=0.54)$.

KEY WORDS: Dynamic, foot, balance, power, speed, tennis, physical therapy.

\section{INTRODUCCIÓN}

La técnica fundamental del tenis se basa en un patrón motor de golpeo, en el que se produce la colisión de dos objetos en movimiento (raqueta y pelota) con diferentes características morfológicas y mecánicas, donde se ponen en juego adaptaciones relacionadas con la cantidad de fuerza, velocidad y sentido del giro de la pelota, y trayectoria a las diversas situaciones del juego. Estas situaciones de juego son acciones que se ejecutan a elevada velocidad y en un corto tiempo (1).

Uno de los determinantes del rendimiento en el tenis es la ejecución del servicio, el cual debe tener gran velocidad y la mayor precisión posible, debido a que este, es el único movimiento en el cual el jugador tiene control total sobre la sincronización, la velocidad, la dirección y la rotación de la pelota, sin ninguna influencia del opositor. Es también uno de los movimientos del gesto deportivo más complejos y requiere años de práctica para realizarlo con eficacia durante una competencia; por este motivo ha sido objeto de investigación (2).

Hablando específicamente de la técnica del servicio de tenis, ésta ha recibido mayor atención en la literatura que los demás golpes, probablemente porque es el golpe más fácil para estudiar, pues se inicia desde una posición fija y el jugador tiene control total. El objetivo principal se centra en la proyección de la pelota con la mayor velocidad posible y en la dirección adecuada para obtener el punto. El servicio 
incluye una sumatoria de fuerzas secuenciadas en gran medida de manera proximal a distal (pies, piernas, tronco, brazo/raqueta); esto requiere una secuencia de movimientos coordinados con el ritmo adecuado de cada segmento (3) (4).

Dentro de la biomecánica del servicio se han encontrado diferencias que podrían ser claves para el análisis de este en los deportistas elite (5), que debido a años de dedicación han logrado obtener mejorías en la mecánica corporal optimizando su desempeño en el campo. Según la literatura se han realizado electromiogramas de la extremidad inferior y han determinado los perfiles de la fuerza de reacción del suelo que caracterizan el servicio de tenis, hallando que el saque de los jugadores de elite se distingue del de los jugadores de nivel inferior por patrones de coordinación neuromuscular más refinados (6).

Estos patrones de coordinación neuromuscular se ven reflejados al utilizar el cuerpo de manera fluida e integral contribuyendo a mejorar la potencia del golpe. Una buena producción de golpes comienza en los pies, fluye por las rodillas y piernas, usa la cadera, depende en parte del peso corporal y permite que la parte superior del cuerpo le impacte la pelota; todo lo anterior dependiente del trabajo de cadenas cinéticas. De tal manera que una buena producción del servicio inicia en la base de sustentación, dado que la transmisión de cargas se produce de manera sincrónica a través de las cadenas musculares que facilitan la producción del gesto, teniendo en cuenta desde la base de sustentación variables como la fuerza de presión del pie, la potencia y centro de masa durante el servicio que pueden llegar a generar mayor comprensión del gesto técnico (7).

Dentro de otras habilidades necesarias para el deporte del tenis en el presente artículo se explorará el equilibrio, la velocidad y la potencia, a su vez los objetivos que se pretenden desarrollar son describir la dinámica plantar durante el servicio y durante la realización de pruebas específicas de potencia, velocidad, equilibrio y correlacionarlos entre sí.

\section{MATERIALES Y MÉTODOS}

Estudio descriptivo realizado en 16 jugadores de tenis de género masculino con promedio de edad de $18( \pm 2,8)$ años y promedio de edad deportiva $7,9( \pm 4,3)$ años, pertenecientes a dos selecciones bogotanas de este deporte. Se excluyeron a los tenistas que hubiesen presentado algún tipo de lesión en el último mes o con síntomas activos de dolor localizado en el hombro, pinzamiento o tendinitis del manguito rotador, dolor irradiado en la columna vertebral, o los miembros inferiores. Se realizaron las mediciones garantizando que los atletas no hubiesen competido en las últimas 48 horas. Todos los participantes firmaron consentimiento informado luego de ser conocedores del riesgo mínimo de la investigación. 


\section{Instrumentos}

Se realizaron pruebas de balance, potencia y velocidad además del servicio. Para la evaluación del balance se utilizó el y test test de la "y"(8) es un test confiable que permite demostrar la simetría funcional. La prueba se realiza manteniendo el equilibrio en una sola pierna, mientras se alcanza lo más lejos posible con la pierna contralateral en tres direcciones diferentes. Las tres direcciones de movimiento son anterior, posteromedial y posterolateral, realizadas en cada pierna. Por lo tanto, hay seis pruebas a realizar, en el siguiente orden: alcance anterior derecho, alcance anterior izquierdo, alcance posteromedial derecho, alcance posteromedial izquierdo, alcance posterolateral derecho y alcance posterolateral Izquierdo, cada uno de los alcances se midió en centímetros.

Para evaluar la potencia se utilizó el test de salto vertical a pie junto, con el siguiente procedimiento: primero se realizó la marcación de altura el ejecutante, quien debía estar de frente a una pared con los pies totalmente apoyados y juntos, el tronco recto y los brazos extendidos por encima de la cabeza, a lo ancho de los hombros, las manos abiertas y con las palmas apoyadas sobre la pared, con el fin de señalar con los dedos medios impregnados de agua, la altura máxima alcanzable. Luego se realizó el salto donde la persona se encontraba lateralmente junto a la pared, a 20 $\mathrm{cm}$ aproximadamente, con el tronco recto, los brazos caídos a lo largo del cuerpo y las piernas extendidas, los pies paralelos a la pared, con una apertura aproximada del ancho de los hombros; a la señal, el ejecutante podía inclinar el tronco, flexionar varias veces las piernas (sin despegar los pies del suelo), y balancear los brazos para realizar un movimiento explosivo de salto hacia arriba. Durante la fase de vuelo, debía extender al máximo el tronco y el brazo más cercano a la pared, marcando con el dedo medio la mayor altura posible. Se midió el número de centímetros que existió entre las dos marcas realizadas por el sujeto. Se consideró inválido el salto que tuviese un giro del tronco. Se realizaron tres intentos y se consideró el mejor para su análisis (9).

Para la evaluación de la velocidad se realizó el Test de 30 metros con salida lanzada. Inicialmente, el deportista se ubicó en posición de salida alta, detrás de una línea situada a $15 \mathrm{~m}$ previa a la línea de cronometraje. A la señal del examinador el ejecutante comenzó a correr lo más rápido posible con el objetivo de alcanzar la máxima velocidad al comenzar los $30 \mathrm{~m}$ cronometrados (señalados con banderines). Se cronometró el tiempo empleado en recorrer los últimos $30 \mathrm{~m}$. El cronometrador se situó en línea de meta, y activó el cronómetro a la señal de un segundo cronometrador situado al inicio de los $30 \mathrm{~m}$, quien bajó el brazo enérgicamente en el instante de pasar el corredor (9), los resultados se registraron en segundos.

Para la evaluación del servicio se utilizó la grabación del jugador posterior a la alternancia de servicios a la zona demarcada por parte de cada jugador (una canasta completa)(10) el tipo de piso en el que se realizaron los servicios fue en polvo de ladrillo. Se realizaron 5 servicios con efecto top spin. 
Para la evaluación de la dinámica plantar se utilizaron las plantillas electrónicas Open-Go MOTICON $\circledast$ (11) durante todas las pruebas, las cuales por medio de 13 sensores miden la distribución de presión en milímetros $(\mathrm{mm})(\mathrm{COP})$, la potencia en Newton sobre segundo(N/s), y la fuerza de reacción del pie en Newton $(\mathrm{N})$ durante cada actividad. La frecuencia de muestreo utilizada fue de $100 \mathrm{~Hz}$. Para entender la dinámica plantar en los tenistas se analizaron algunas variables según las pruebas aplicadas.

Durante la prueba de equilibrio "y" test se analizó el COP $\mathrm{mm}$ en el plano anteroposterior (AP) y mediolateral (ML) del pie, además de la velocidad del COP en $\mathrm{mm} / \mathrm{ms}$ durante las seis posiciones. Durante la prueba de potencia se tuvo en cuenta el promedio del tiempo en el aire determinado por los sensores de las plantillas. En la prueba de velocidad se analizó el tiempo del ciclo de la carrera y por último las variables analizadas de la dinámica plantar durante el servicio fueron el promedio de tiempo en el aire del salto que se realiza en este servicio (efecto top spin). Además, se analizó la potencia del salto en N/s, la presión plantar en N/cm2 durante el despegue del salto y durante el aterrizaje y finalmente la fuerza de reacción en $\mathrm{N}$ de la pierna derecha e izquierda.

\section{Procedimiento}

Luego de realizar la firma del consentimiento informado se realizó un calentamiento global y específico posteriormente se posicionaron las plantillas calibrando la presión de cero en cada jugador, seguido de ello, se realizaron las pruebas de equilibrio, potencia y velocidad y al final se realizó el calentamiento específico de los servicios (una canasta) para terminar con la grabación de los cinco últimos servicios. Se retiraron las plantillas y se descargaron los datos de cada jugador para su depuración y análisis.

\section{Análisis}

Se registraron todos los datos en el procesador Excel Microsoft $\AA$, se realizaron medidas descriptivas de promedio y desviación estándar, además se establecieron medidas de correlación de Sperman entre los resultados de las pruebas físicas y la dinámica plantar medida con las plantillas Open Go MOTICON ${ }^{\circledR}$.

\section{RESULTADOS}

Los participantes evaluados tuvieron un índice de masa corporal promedio de 21,5 $\pm 3,4$. Dentro de las pruebas realizadas (Tabla 1.) los centímetros saltados fueron en promedio $47,6 \pm 6,2 \mathrm{~cm}$, el tiempo promedio en recorrer los 30 metros fueron 3,8 seg, y los centímetros alcanzados en el "y" test con la pierna contralateral al apoyo fueron cercanos para ambos miembros inferiores. 
Tabla 1. Descriptivos Pruebas Físicas

\begin{tabular}{|c|c|c|}
\hline PRUEBA FÍSICA & PROMEDIO & DS \\
\hline \multicolumn{2}{|c|}{ EQUILIBRIO MID (cm) } \\
\hline ANTERIOR DERECHA & 60,8 & 6,37 \\
\hline POSTERIOR DERECHA & 94,8 & 12,5 \\
\hline POSTERIOR IZQUIERDA & 85,8 & 11,0 \\
\hline \multicolumn{3}{|c|}{ EQUILIBRIO MII (cm) } \\
\hline ANTERIOR DERECHA & 63,5 & 5,8 \\
\hline POSTERIOR DERECHA & 94,8 & 12,5 \\
\hline POSTERIOR IZQUIERDA & 86,7 & 11,6 \\
\hline SALTO VERTICAL (cm) & 47,6 & 6,2 \\
\hline VELOCIDAD (s) & 3,8 & 0,5 \\
\hline
\end{tabular}

MID: Miembro inferior derecho

MII: Miembro inferior izquierdo

Con respecto a la dinámica plantar durante la prueba de equilibrio fue evidente que el COP AM se desplaza más hacia posterior en el pie derecho que en el izquierdo, a su vez la velocidad del COP es mayor en la pierna izquierda.

En la (Figura 1) se observa el comportamiento del centro de presión de uno de los deportistas en un plano cartesiano, en el pie derecho (color verde) y el pie izquierdo (color rojo) representan el pie de apoyo en la prueba del Y test, la zona marcada con color indica el desplazamiento del centro de presión en sentido anteroposterior sobre el eje $\mathrm{Y}$, se observa que el comportamiento es similar en ambos pies.

Fig. 1 Desplazamiento del centro de presión en el "Y" test

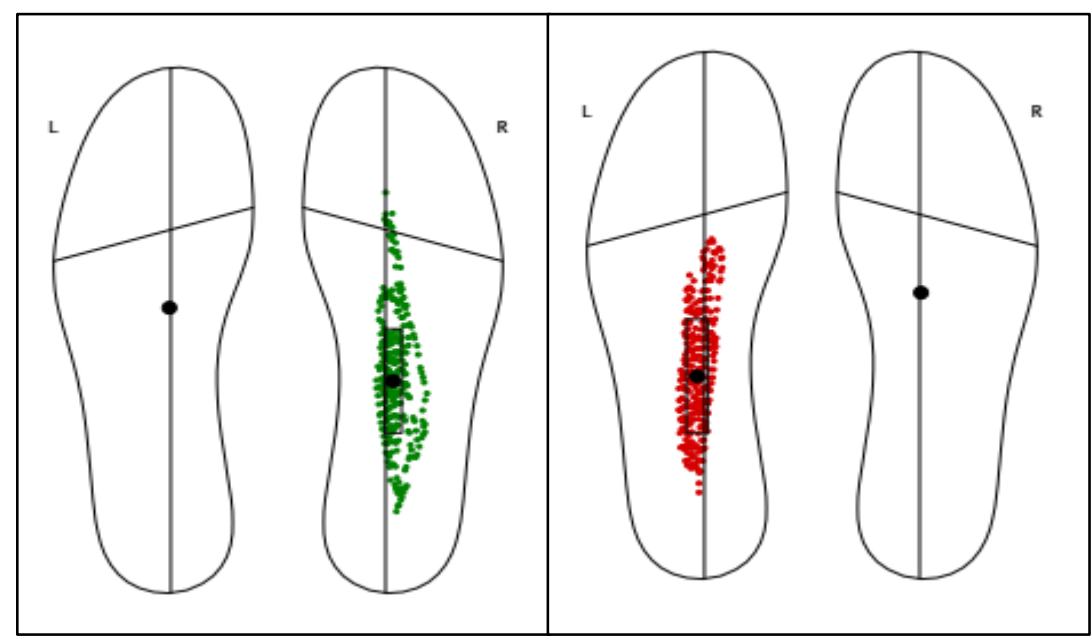

Con respecto al salto se encontró un promedio de tiempo en el aire de 0,57 segundos y una potencia en el salto de $165 \mathrm{~N}$. Con respecto a la presión fue mayor en el momento del aterrizaje, y la fuerza de reacción en el suelo siempre fue mayor en la pierna izquierda (Tabla 2). 
Tabla 2. Descriptivos de la Dinámica Plantar en Cada Test

\begin{tabular}{|l|c|c|}
\hline EQUILIBRIO (Y TEST) & DERECHA & IZQUIERDA \\
\hline COP AM(mm) & $-32,5(31,7)$ & $-11,35(26,4)$ \\
\hline COP ML $(\mathrm{mm})$ & $-0,29(1,7)$ & $0,41(2,9)$ \\
\hline VELOCIDAD COP mm/ms & $235,9(92,8)$ & $247,5(71,1)$ \\
\hline SALTO VERTICAL & $0,53(0,15)$ \\
\hline TIEMPO EN EL AIRE (s) & \multicolumn{2}{|c|}{$0,52(0,26)$} \\
\hline VELOCIDAD (30m Lanzados) & \multicolumn{2}{|c|}{$0,57(0,28)$} \\
\hline TIEMPO DEL CICLO DE LA CARRERA & \multicolumn{1}{|c|}{$165(35)$} \\
\hline SERVICIO & $41(1,4)$ & $52,6(2,0)$ \\
\hline TIEMPO EN EL AIRE (s) & $69,4(2,4)$ & $65,1(2,8)$ \\
\hline POTENCIA DEL SALTO N/s & $114(20,5)$ & $122(21,8)$ \\
\hline PRESIÓN DURANTE EL DESPEGUE (N/cm2)
\end{tabular}

Al establecer la correlación entre los resultados de la dinámica plantar obtenidos por medio del Software Open Go y los datos por cada una de las pruebas se encontró una relación moderada entre el desplazamiento del COP ML y el alcance posterior de la pierna izquierda al evaluar el equilibrio de la pierna derecha $(p<0,05)$. Igualmente se encontró una relación entre la velocidad del COP y los centímetros de alcance posterior de la misma pierna (Tabla 3). 
Rev.int.med.cienc.act.fís.deporte - vol. 19 - número 75 - ISSN: 1577-0354

Tabla 3. Índices de Correlación Entre Dinámica Plantar y Pruebas Físicas

\begin{tabular}{|c|c|c|c|}
\hline PRUEBA FÍSICA & COP AP(mm) & COP ML(mm) & $\begin{array}{l}\text { VELOCIDAD } \\
\text { COP } \mathrm{mm} / \mathrm{ms}\end{array}$ \\
\hline \multicolumn{4}{|l|}{ EQUILIBRIO MID (cm) } \\
\hline ANTERIOR DERECHA & $-0,137608405$ & $0,381667806^{*}$ & 0,077952671 \\
\hline POSTERIOR DERECHA & 0,167775502 & $-0,205998346$ & 0,259409963 \\
\hline POSTERIOR IZQUIERDA & 0,104870279 & $-0,224225519$ & $0,396900244^{*}$ \\
\hline \multicolumn{4}{|l|}{ EQUILIBRIO MII (cm) } \\
\hline ANTERIOR DERECHA & $-0,006555578$ & $-0,138853951$ & $-0,089117244$ \\
\hline POSTERIOR DERECHA & $-0,137172303$ & $-0,203107556$ & 0,077510609 \\
\hline POSTERIOR IZQUIERDA & $-0,222391818$ & $-0,1841351$ & 0,29023066 \\
\hline \multirow{2}{*}{ SALTO VERTICAL (cm) } & \multicolumn{3}{|c|}{ TIEMPO EN EL AIRE } \\
\hline & \multicolumn{3}{|c|}{$0,452012858^{*}$} \\
\hline \multirow{4}{*}{ VELOCIDAD (s) } & \multicolumn{3}{|c|}{ TIEMPO DEL CICLO DE LA CARRERA } \\
\hline & \multicolumn{3}{|c|}{$0,525994415^{\star}$} \\
\hline & \multicolumn{3}{|c|}{ TIEMPO DE DOBLE SOPORTE } \\
\hline & \multicolumn{3}{|c|}{0,029120034} \\
\hline
\end{tabular}

También se encontró una relación entre el tiempo en el aire durante el salto vertical y los centímetros alcanzados en la prueba. Finalmente se encontró una relación entre los segundos de la prueba de 30 metros lanzados con el tiempo del ciclo de la carrera reportado en el software.

\section{DISCUSIÓN}

Los hallazgos encontrados al analizar las variables de presión plantar, potencia y COP en tenistas durante movimientos seleccionados relacionados con el deporte de tenis, incluyendo saltos, velocidad y equilibrio muestran que el COP tiende a ser más posterior en la pierna derecha, además la velocidad del COP fue menor en ese miembro inferior pudiéndose relacionar un COP con tendencia hacia posterior con mayor equilibrio en la prueba aplicada y menor desplazamiento del COP. Además, la presión plantar fue mayor durante el aterrizaje que el despegue y el miembro inferior donde mayor fuerza de presión se generó fue el izquierdo.

Teniendo en cuenta lo anterior, investigaciones donde se ha delimitado la presión plantar como variable en relación con el pie dominante en otros deportes como el fútbol y el atletismo, han demostrado que la distribución de las presiones plantares en los participantes es homogénea en ambos pies en un gesto que implica tantas activaciones de cadenas musculares; esta distribución ha sido analizada con herramientas como las plantillas EMED Pedar de la compañía Novel, y el sistema de plantillas Footscan (12)(13). No obstante, las plantillas descritas anteriormente, 
sólo miden la presión plantar (14), mientras que las plantillas Open Go Physio, utilizadas en el trabajo actual permiten medir la distribución de presiones plantares, cargas totales y trayectoria del centro de gravedad de manera simultánea, precisa e inalámbrica (11).

Es importante resaltar que las presiones plantares se ven influenciadas por la interacción del deportista con el entorno en términos de calzado deportivo específico, el tipo de movimiento, impulso y/o la naturaleza de la superficie del suelo (cancha), lo que puede modificar la forma en que se distribuye la carga y se desplaza el centro de masa (3); esto dificulta la comparación directa de presiones plantares con otros estudios como los desarrollados con las plantillas EMED Pedar y Footscan donde el análisis de estas presiones se realizaron en un ambiente controlado, debido a que no son inalámbricas, pues no permiten el desplazamiento a otros campos(12)(13). Por el contrario, las plantillas Open Go Physio, permiten la toma de datos de variables en el campo de juego con superficie de polvo de ladrillo y cancha dura, que son específicas para el juego de tenis, como fue el caso del presente trabajo (16).

Por otra parte, hay otros estudios como el realizado por Girard (17) sobre análisis de presiones plantares durante el servicio de tenis con uso de técnicas electromiografías, el cual concluyó que el $80 \%$ de la carga total del pie delantero se concentró en el hallux, los dedos de los pies y el ante pie, lo que sugiere que la zona anterior del pie delantero puede contribuir significativamente al buen desempeño en el servicio; mientras que por otro lado en el pie posterior, las cargas relativas se distribuyen a través del pie de manera similar y con una menor variabilidad. Esto es concordante con los resultados obtenidos en el presente trabajo donde se evidenció que el desplazamiento del centro de masa de los participantes en ambos grupos fue mayor hacia la parte anterior del pie que hacia la posterior del mismo en la fase del salto durante el servicio, con valores positivos en el análisis obtenido con el uso de las plantillas Open GO Physio.

Es necesario realizar investigaciones con mayor tamaño de muestra que permita comprender el comportamiento de las fuerzas de presión durante el servicio además de mejor comprensión de la dinámica plantar en general en el tenis.

\section{CONCLUSIONES}

Durante el servicio tipo top spin se encontró mayor fuerza de presión en la fase de aterrizaje. En la fase de despegue el pie que recibió más carga fue el izquierdo; en cambio, durante el aterrizaje el pie que recibió mayor presión por centímetro cuadrado fue el derecho.

\section{REFERENCIAS BIBLIOGRÁFICAS}

1. Frutos JB. Análisis estructural del golpeo en tenis: una aproximación desde la biomecánica. Eur J Hum Mov. 2013;(31):111-33. 
2. Infantes TU, Davó HH, Hernández FJM. Variabilidad cinemática en relación con el rendimiento en el saque en jóvenes tenistas. Eur J Hum Mov. 2012;(29):49-60.

3. Izquierdo M, Redín M. Biomecánica y bases neuromusculares de la actividad física y el deporte. Madrid: Ed. Médica Panamericana; 2008. 796 p.

4. Gómez M, Zissu M. Evaluación biomecánica del saque de tenis de campo ejecutado por atletas participantes en el Torneo Internacional Future. Dir Aula Competencia Med y Ciencias Apl. 2006;22.

5. Menayo R, García JPF, Hernández FJM, Clemente R, Calvo TG. Relación entre la velocidad de la pelota y la precisión en el servicio plano en tenis en jugadores de perfeccionamiento. Eur J Hum Mov. 2008;(21):17-30.

6. Ellenbecker TS, Roetert EP, Reid M. Biomecánica del Saque de Tenis: Consecuencias para el Entrenamiento de Fuerza. Strength and Conditioning Journal. 2009; 31 (4):35-40 https://doi.org/10.1519/SSC.0b013e3181af65e1

7. Abrams GD, Harris AH, Andriacchi TP, Safran MR. Biomechanical analysis of three tennis serve types using a markerless system. Br J Sports Med. 2014 Feb;48(4):339-42. https://doi.org/10.1136/bjsports-2012-091371

8. Shaffer SW, Teyhen DS, Lorenson CL, Warren RL, Koreerat CM, Straseske CA, et al. Y-Balance Test: A Reliability Study Involving Multiple Raters. Mil Med. 2013 Nov;178(11):1264-70. https://doi.org/10.7205/MILMED-D-13-00222

9. López E. Pruebas de aptitud física. Barcelona: Editorial Paidotribo; 2002. 370 p.

10. Álvarez J. Calentamiento y vuelta a la calma en el tenis. Revista digital Editorial Universitas MH; $2011 . \quad$ Disponible en https://www.yumpu.com/es/document/view/14367978/alonso-j-espcalentamiento-y-vuelta-a-la-calma-en-el-tenis-itf.

11. Stöggl T, Martiner A. Validation of Moticon's OpenGo sensor insoles during gait, jumps, balance and cross-country skiing specific imitation movements. J Sports Sci. 2017;35(2):196-206. https://doi.org/10.1080/02640414.2016.1161205

12. Zafra $P$, Berna J. Análisis de la presión plantar entre el pie dominante y no dominante en jugadores de fútbol profesional. THERAPEIA 6. 2014: 45-57

13. Hong $\mathrm{Y}$, Wang $\mathrm{L}$, Li JX, Zhou JH. Comparison of plantar loads during treadmill and overground running. J Sci Med Sport. 2012 Nov;15(6):554-60. https://doi.org/10.1016/i.jsams.2012.01.004

14. García F. Reflexiones en Torno al Movimiento Corporal Humano desde una Perspectiva Multidimensional y Compleja. Ciencias e innovación en salud. 2013; 1(1):78-91 https://doi.org/10.17081/innosa.1.1.88

15. Natali AN, Forestiero A, Carniel EL, Pavan PG, Dal Zovo C. Investigation of foot plantar pressure: experimental and numerical analysis. Med Biol Eng Comput. 2010 Dec;48(12):1167-74. https://doi.org/10.1007/s11517-010-0709-8

16. Girard O, Eicher F, Micallef J-P, Millet G. Plantar pressures in the tennis serve. J Sports Sci. 2010; 28(8):873-80. https://doi.org/10.1080/02640411003792695

Número de citas totales / Total references: 16 (100\%) Número de citas propias de la revista /Journal's own references: 0 
Rev.int.med.cienc.act.fís.deporte - vol. 19 - número 75 - ISSN: 1577-0354

Rev.int.med.cienc.act.fís.deporte - vol. 19 - número 75 - ISSN: 1577-0354 